\title{
Successful Excision of a Right Ventricular Fibroma Associated with Ventricular Tachycardia

\author{
Report of a Six Year Survival
}

\author{
Michio Kimura, M.D.,* Takehiko Fujino, M.D.,** \\ Yoshito KawachI, M.D.,*** and Katsushi Kitazato, M.D.****
}

\begin{abstract}
SUMmary
A right ventricular (RV) tumor manifested by ventricular tachycardia (VT) accompanied by syncopal attacks was found in a 14 year old boy by two-dimensional echocardiography. Surgery was performed on February 10, 1981, with the aid of a cardiopulmonary bypass. The tumor was completely removed as a mass from the anterolateral portion of the RV wall. The wall was closed directly without any patch. The mass was $60 \mathrm{gm}$ in weight and 7 by 4 by $4 \mathrm{~cm}$ in size. Fibroma was diagnosed by pathological study. The patient is doing well 6 years postoperatively. We conclude that two-dimensional echocardiography should be used to exclude cardiac tumors such as fibroma in young patients who have VT.
\end{abstract}

\section{Additional Indexing Words:}

Right ventricular (RV) fibroma

Ventricular tachycardia (VT)

Two-dimensional echocardiography

\begin{abstract}
A
RV fibroma which manifested as VT with syncopal attacks was successfully removed from a 14 year old boy. Two-dimensional echocardiography revealed a mass in the RV and this finding led the patient to receive surgical treatment. He is doing well with no evidence of recurrence and is free from VT 6 years postoperatively.

Reports of long-term survivors after excision of cardiac fibromas are few $^{1), 2)}$ and furthermore, VT has rarely been reported as the manifestation of a RV fibroma. ${ }^{3), 4)}$

In this paper, VT as a dangerous manifestation of a RV fibroma and a 6 year follow-up after successful excision are discussed.

From the Department of Cardiovascular Surgery, Fukuoka University School of Medicine,* First Department of Internal Medicine, Kyushu University School of Medicine, ${ }^{*}$ Department of Cardiovascular Surgery, Kyushu University School of Medicine, ${ }^{* * *}$ and Department of Cardiovascular Surgery, National Fukuoka Higashi Hospital,**** Fukuoka.

Address for reprints: Michio Kimura, M.D., Department of Gardiovascular Surgery, Fukuoka University School of Medicine, 7-45-1 Nanakuma, Jyonan-ku, Fukuoka City 814-01, Japan.

Received for publication December 27, 1986.

Accepted April 11, 1988.
\end{abstract}




\section{CAse Report}

A 14 year old boy was admitted to the National Fukuoka Higashi Hospital on January 20, 1981, with the chief complaint of syncopal attack due to VT (Fig. 1-A). DG conversion was immediately done. Electrocardiography taken after the conversion showed complete right bundle branch block (Fig. 1-B). Prior to this admission, ventricular premature contraction and VT had been detected at other hospitals since his childhood but neither special examination nor treatment had been given.

At the first examination in this admission, an abnormal mass in the heart was not diagnosed although M-mode echocardiography was performed. On January 23, two-dimensional echocardiography with a Toshiba Sonolayer SSL 53-MS revealed a mass in the RV wall moving synchronously with the heart beat as shown in Fig. 2.

On January 27, cardiac blood pool imaging using ${ }^{99 m}$ Tc-HSA was done. In the left anterior oblique position, a narrowing of the RV cavity during

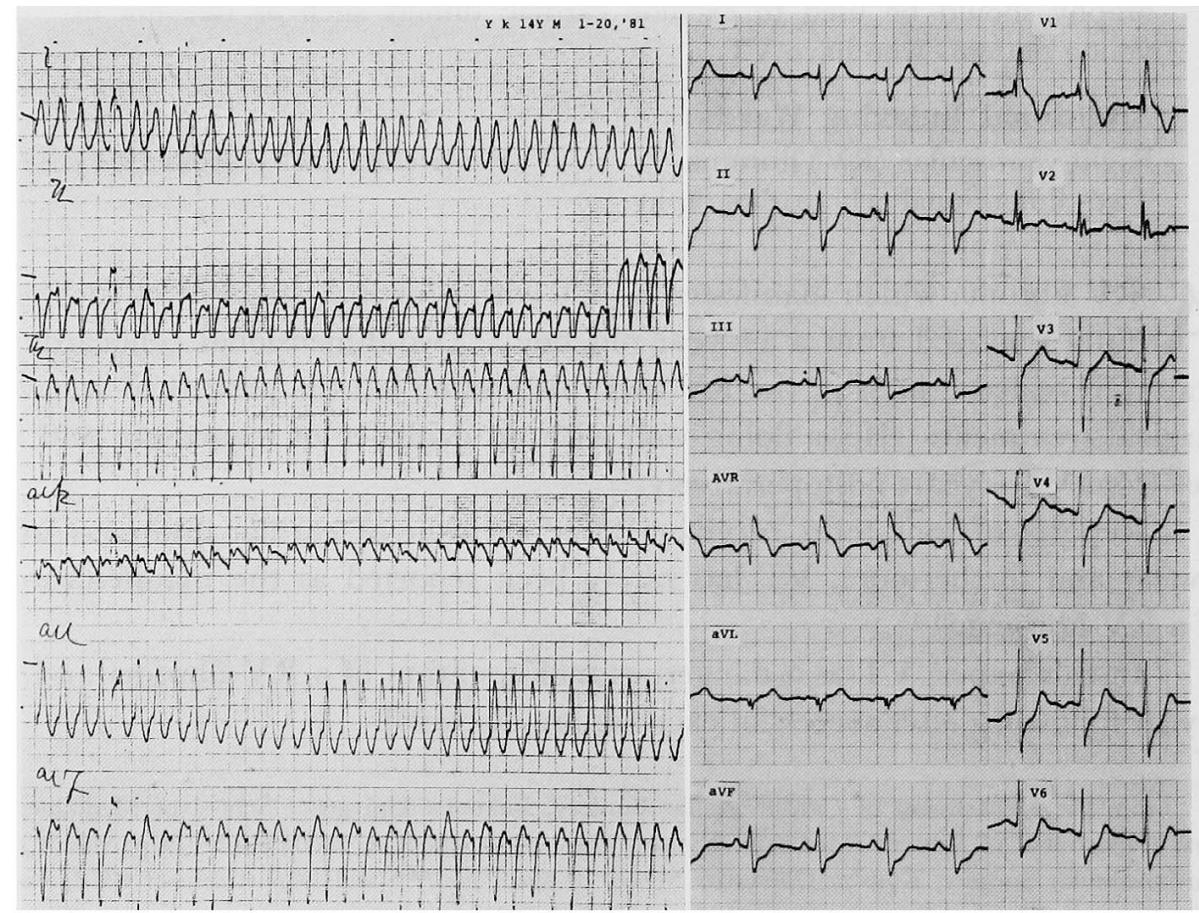

(A)

(B)

Fig. 1. Electrocardiogram at admission showed ventricular tachycardia (A, left). After DG conversion, ordinary sinus rhythm was restored (B, right). 
systole was seen.

Cardiac catheterization and right ventriculography were done on January 28. There was no significant oxygen step-up in any chambers in the right heart. No pressure gradient was observed between the pulmonary artery and the RV, in the RV itself and between the RV and the right atrium. Cineangiography showed a negative shadow of moderate size near the RV inflow moving synchronously with the heart beat. There was no obstruction in the RV or pulmonary artery.

Routine blood chemistry and urinalysis showed no abnormal findings.

The patient had surgery on February 10, 1981. Median sternotomy was carried out. No fluid had accumulated in the pericardial cavity and no adhesion was seen between the heart and the pericardium. In the anterior wall of the RV, a mass covered by the epicardium was palpated. It occupied about one third of the RV anterior wall, located between the outflow
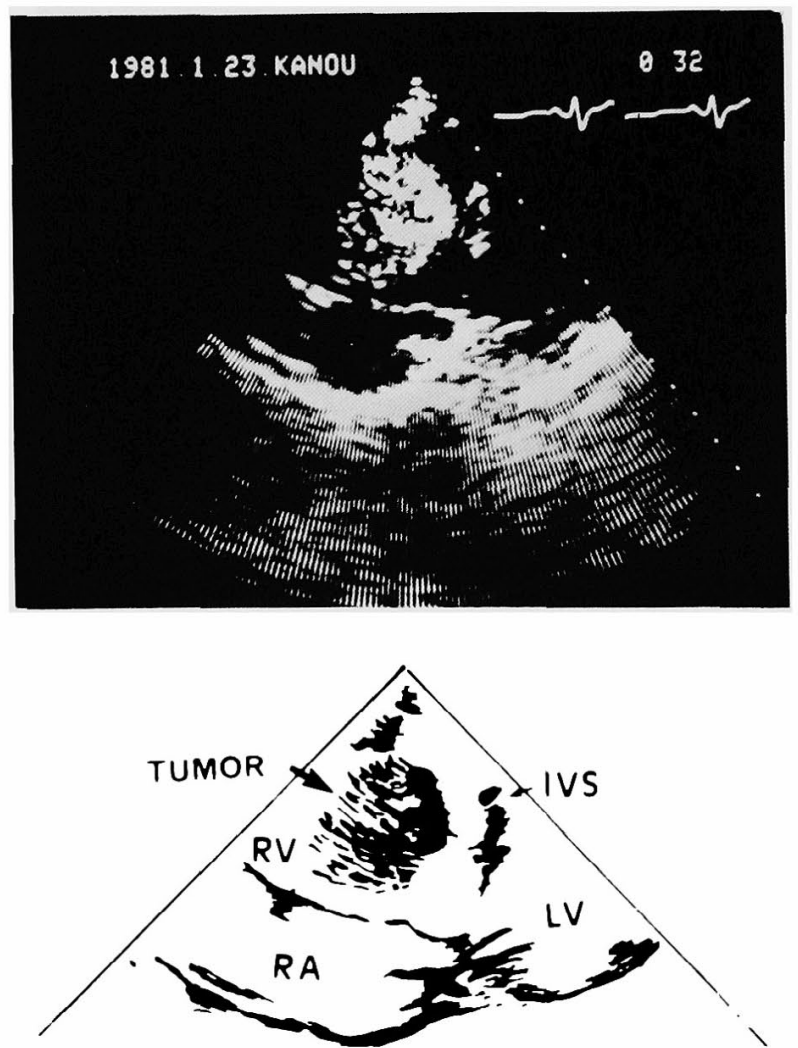

Fig. 2. Two-dimensional echocardiogram by modified parasternal four chamber view and the schema before the operation. RV-mass is shown by arrow. 


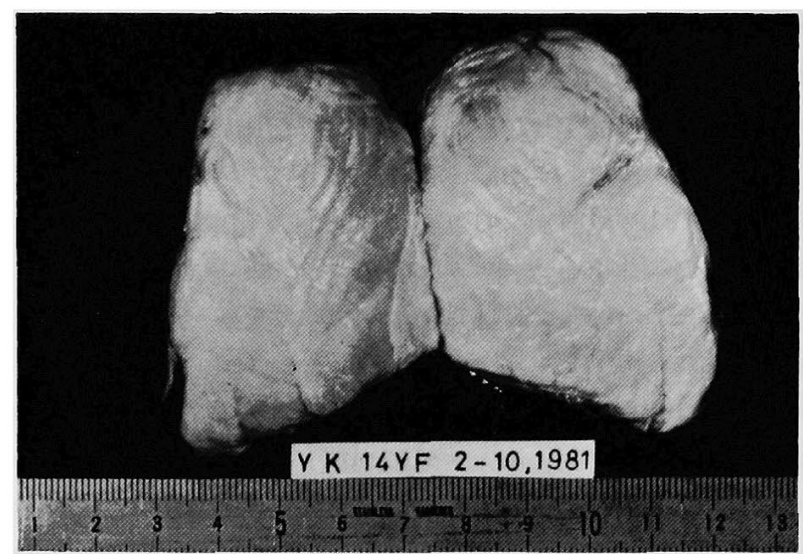

Fig. 3. The cut surface of the excised tumor. Pathological study revealed it to be a fibroma.

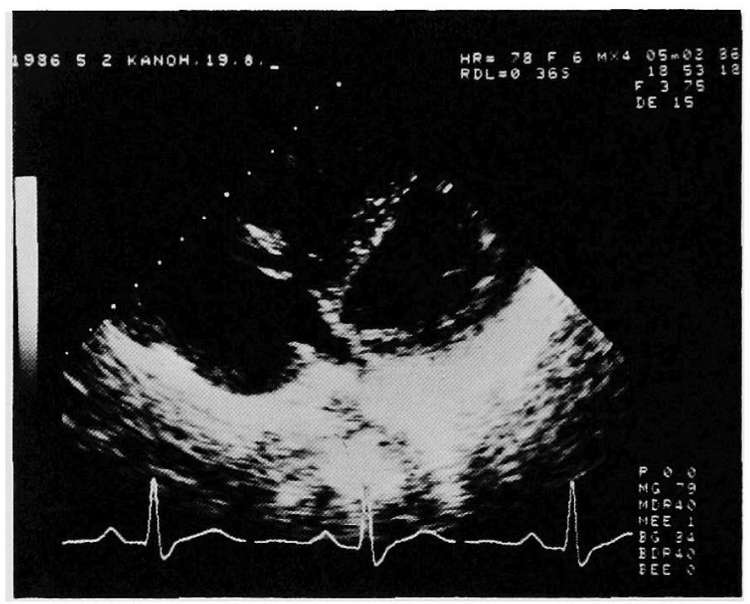

Fig. 4. Two-dimensional echocardiogram by modified parasternal four chamber view 6 years postoperatively. It shows no recurrence of the tumor.

portion of the RV and the acute marginal artery. The upper border did not extend past the right atrioventricular sulcus. The lower margin was close to the RV apex. Ordinary cardiopulmonary bypass was established. The heart was arrested by aortic cross-clamping and cold potassium cardioplegia. Topical ice slush cooling was also applied. The RV anterior wall was incised along the medial border of the tumor. The RV cavity was entered. The tricuspid valve and the RV cavity were intact. The tumor was removed by further incision running parallel to the atrioventricular sulcus and along the medial border of the acute marginal coronary artery. It was not encapsulated and cardiac muscles were partially attached. The incised RV wall was 
closed directly. No narrowing of the RV cavity resulted from this procedure. The patient was able to sustain this procedure and recovered from anesthesia.

The tumor was as large as 7 by 4 by $4 \mathrm{~cm}$ in size and $60 \mathrm{gm}$ in weight. The cut surface was gray white in color and of a rubbery consistency. No calcification was seen (Fig. 3). Pathological study revealed that it was a fibroma.

The postoperative course was quite event free and the patient was discharged on March 19, 1981. Six years later, he is doing well without any suspicion of recurrence of the tumor and is free of arrhythmia. Two-dimensional echocardiography after 6 years showed no abnormal findings (Fig. 4).

\section{Discussion}

It is reported that arrhythmia is found in association with cardiac fibroma in approximately $30 \%$ of cases, ${ }^{5}$ and sudden death occurs without any previous symptoms in about one fourth of reported cases, ${ }^{6)}$ but there are few reports concerning VT in such cases. ${ }^{3), 4)}$ There is a report that cardiac arrest following VT was seen in a two and a half year old boy with a RV fibroma. $^{3)}$ This patient was resuscitated and operated on soon after recovery but died due to low cardiac output syndrome. Another 2 cases of cardiac fibromas accompanied by VT have been reported.4) In both, although the tumor was located in the left ventricle complete excision resulted in resolution of VT. In these 3 cases, it seemed that the tumors had no direct connection with the conduction system because they were located in the ventricular free wall, not in the interventricular septum. Thus, occurrence of tachyarrhythmias rather than bradyarrhythmias may be considered. The mechanism of VT is not clearly explained but the existence of the tumor in the ventricular wall may result in the creation of an irritable focus or vulnerability to a reentry rhythm.

Resolution of VT after excision of the tumors reported in the literature ${ }^{6)}$ and also in our case may suggest the rationale for the hypothesis previously mentioned. In another point of view, when VT is seen in younger patients, one must consider the existence of a cardiac tumor as the cause of this severe arrhythmia.

Two-dimensional echocardiography was particularly effective for detection of the cardiac tumor. In this patient, M-mode echocardiography was applied at first but no mass was found. By two-dimensional echocardiography a tumor in the $\mathrm{RV}$ wall was revealed. Ports et $\mathrm{al}^{\text {7) }}$ recommended the use of two-dimensional echocardiography to complete M-mode techniques 
for noninvasive diagnosis of a RV tumor. As to the accuracy of this examination technique, detectable size of the tumor varies from as small as $1 \mathrm{~cm}$ to a large mass. Furthermore, abnormal motions of the tumor which cause outflow obstruction in the cardiac chambers are detectable. ${ }^{81,9)}$ On the other hand, Moraes et $\mathrm{al}^{10}$ ) stated that echocardiography could not detect a left ventricular fibroma. This means echocardiography is not absolutely satisfactory in diagnosing cardiac tumors. Combining it with an isotope study may give further information. ${ }^{11), 12)}$ In this case, radionuclide blood pool scanning was done additionally and narrowing of the RV cavity was shown, but the size and extent of the tumor was not clear. In this case, two-dimensional echocardiography was quite effective compared with other examinations.

The principle of surgical treatment for cardiac fibromas is complete excision. To follow the postoperative course is important because of the possibility of recurrence of the tumor. As to long-term survival after excision, to our knowledge, by 1987 , few reports had been made. ${ }^{1,2)}$ Williams et al ${ }^{11}$ reported 3 cases of long-term survivors followed-up 13, 15 and 17 years after excision. They are reported doing well but one of them (Case 3 ) is thought to have a residual tumor and signs of mitral stenosis. Reece et $\mathrm{al}^{2}{ }^{2}$ reported 2 patients surviving 10 and 18 years. They are asymptomatic but incomplete excision had been done in both. According to these reports, complete excision is desirable but long-term survival is expected even in the case of incomplete excision. Incompletely excised cases are those where the tumor is in close relationship with the interventricular septum and the coronary artery. In our case, the location of the tumor was the anterior wall of the RV and consequently there was no close relationship with the coronary artery, the interventricular septum or the tricuspid valve apparatus. Thus, complete excision was done. No recurrence of the tumor was seen on two-dimensional echocardiography, nor has VT been observed 6 years following surgery. Although further follow-up is necessary, long-term survival is expected in this patient.

\section{ACKNOWLEDGMENTS}

We thank Katsuo Sueishi, M.D., Professor of Pathology, Kyushu University School of Medicine, and Yukihisa Nagabuchi, M.D., Kyushu Chuo Hospital, for the pathological study, and Tsutomu Takemura, M.D., for introducing the patient.

\section{REFERENGES}

1. Williams DB, Danielson GK, McGoon DC, Feldt RH, Edwards WD: Cardiac fibroma. 
Long-term survival after excision. J Thorac Cardiovasc Surg 84: 230, 1982

2. Reece IJ, Cooley DA, Frazier OH, Hallman GL, Powers PL, Montero GG: Cardiac tumors. Clinical spectrum and prognosis of lesions other than classical benign myxoma in 20 patients. J Thorac Cardiovasc Surg 88: 439, 1984

3. Simcha A, Wclls BG, Tynan MJ, Waterston DJ: Primary cardiac tumors in childhood. Arch Dis Child 46: 508, 1971

4. Engle MA, Ebert PA, Redo SF: Recurrent ventricular tachycardia due to resectable cardiac tumor. Report of two cases in two years olds in heart failure. Circulation 50: 1052, 1974

5. Aryanpur I, Nazarian I, Razmara M, Sheikh MA, Khonsari S: Calcified right ventricular fibroma causing outflow obstruction. Am J Dis Child 130: 1265, 1976

6. Geha AS, Weidman WH, Soule EH, McGoon DC: Intramural ventricular cardiac fibroma. Successful removal in two cases and review of the literature. Circulation 36: 427, 1967

7. Ports TA, Schiller NB, Strunk BL: Echocardiography of right ventricular tumors. Circulation 56: 439, 1977

8. Nanda NG, Barold SS, Gramiak R, Ong LS, Heinle RA: Echocardiographic features of right ventricular outflow tumor prolapsing into the pulmonary artery. Am J Cardiol 40: 272, 1977

9. Melendez LJ, Sears GA, Coles JG: Right ventricular tumor demonstrated by echocardiography. Can Med Assoc J 118: 62, 1978

10. Moraes GR, Victor EG, Moscoso J, Lira V, Lima R, Rodrigues JV: Successful excision of an intramural fibroma of the left ventricle. J Cardiovasc Surg 24: 256, 1983

11. Caralis DG, Kennedy HL, Bailey I, Bulkley BH: Primary right cardiac tumor. Detection by echocardiographic and radioisotopic studies. Chest 77: 100, 1980

12. Pitcher D, Wainwright R, Brennand-Roper D, Deverall P, Sowton E, Maisey M: Cardiac tumours: non-invasive detection and assessment by gated cardiac blood pool radionuclide imaging. Br Heart J 44: 143, 1980 